\title{
Robust Model Predictive Control Using Linear Matrix Inequalities for the Treatment of Asymmetric Output Constraints
}

\author{
Mariana Santos Matos Cavalca, ${ }^{1}$ Roberto Kawakami Harrop Galvão, ${ }^{2}$ \\ and Takashi Yoneyama \\ ${ }^{1}$ Departamento de Engenharia Elétrica, Centro de Ciências Tecnológicas, Universidade do Estado de Santa Catarina, \\ Praça Rua Paulo Malschitzki, Zona Industrial Norte, 89.219-710 Joinville, SC, Brazil \\ ${ }^{2}$ Divisão de Engenharia Eletrônica, Instituto Tecnológico de Aeronáutica, Praça Marechal Eduardo Gomes, 50 Vila das Acácias, \\ 12.228-900 São José dos Campos, SP, Brazil
}

Correspondence should be addressed to Mariana Santos Matos Cavalca, mariana.smcavalca@gmail.com

Received 30 June 2011; Revised 22 September 2011; Accepted 6 October 2011

Academic Editor: Marcin T. Cychowski

Copyright ( $) 2012$ Mariana Santos Matos Cavalca et al. This is an open access article distributed under the Creative Commons Attribution License, which permits unrestricted use, distribution, and reproduction in any medium, provided the original work is properly cited.

\begin{abstract}
One of the main advantages of predictive control approaches is the capability of dealing explicitly with constraints on the manipulated and output variables. However, if the predictive control formulation does not consider model uncertainties, then the constraint satisfaction may be compromised. A solution for this inconvenience is to use robust model predictive control (RMPC) strategies based on linear matrix inequalities (LMIs). However, LMI-based RMPC formulations typically consider only symmetric constraints. This paper proposes a method based on pseudoreferences to treat asymmetric output constraints in integrating SISO systems. Such technique guarantees robust constraint satisfaction and convergence of the state to the desired equilibrium point. A case study using numerical simulation indicates that satisfactory results can be achieved.
\end{abstract}

\section{Introduction}

Model-based predictive control (MPC) is a strategy in which a sequence of control actions is obtained by minimizing a cost function considering the predictions of a process model within a certain prediction horizon. At each sample time, only the first value of this sequence is applied to the plant, and the optimization is repeated in order to use feedback information $[1,2]$. One of the main advantages of MPC is the possibility to consider explicitly the physical and operational constraints of the system during the design of the control loop [1-3]. However, if there are mismatches between the nominal model and the actual behavior of the process, then the performance of the control loop can be degraded and the optimization problem may even become unfeasible. Thus, the study of new strategies for design of robust MPC (RMPC) with guaranteed stability and constraint satisfaction properties even in the presence of uncertainties is an area with great potential for research [4-7].
In this context, Kothare et al. [7] proposed an RMPC strategy with infinite horizon employing linear matrix inequalities (LMIs) for dealing with model uncertainty and symmetric constraints on the manipulated and output variables. This approach was later extended to encompass multimodel representations $[8,9]$, setpoint management [10], integrator resetting [11], and offline solutions [12, 13].

Within this scope, Cavalca et al. [4] proposed a heuristic procedure that allows the inclusion of asymmetric constraints on the plant output, but without stability or constraint satisfaction guarantees. The present paper presents a formal strategy to handle asymmetric output constraints in the control of integrating single input, single output (SISO) systems, for the case where the output is linear in states. It is shown that robust constraint satisfaction is achieved, as well as convergence of the state trajectory to the desired equilibrium point. The effectiveness of the proposed method is illustrated by means of numerical simulations. 
The remainder of this paper is organized as follows. Section 2 reviews the design of RMPC based on LMI. Section 3 formalizes the proposed technique for the inclusion of asymmetric constraints. Section 4 presents a case study consisting of a discrete time model of a double integrator. The results are evaluated through numerical simulations as presented in Section 5. Concluding remarks are presented in Section 6.

Throughout the text, I represents an identity matrix, the notation $(\cdot \mid k)$ is used in predictions with respect to time $k$, and the $*$ superscript indicates an optimal solution. For brevity of notation, only the upper triangular part of symmetric matrices is explicitly presented.

\section{LMI-Based RMPC}

Consider a linear time-invariant system described by an uncertain model of the following form:

$$
\begin{gathered}
x(k+1)=A x(k)+B u(k), \\
y(k)=C x(k),
\end{gathered}
$$

where $x(k) \in \mathbb{R}^{n}, u(k) \in \mathbb{R}^{p}$, and $y(k) \in \mathbb{R}^{q}$ are the states, the manipulated and the output variables, respectively, for each time $k$, and $A, B$, and $C$ are constant matrices of appropriate dimensions. The uncertainty is represented in polytopic form; that is, matrices $A$ and $B$ are unknown to the designer, but they are assumed to belong to a convex polytope $\Omega$ with $L$ known vertices $\left(A_{i}, B_{i}\right), i=1,2, \ldots, L$, so that $[2,7]$ :

$$
(A, B)=\sum_{i=1}^{L} \lambda_{i}\left(A_{i}, B_{i}\right)
$$

for some unknown set of coefficients $\lambda_{1}, \lambda_{2}, \ldots, \lambda_{L}$ that satisfy

$$
\sum_{i=1}^{L} \lambda_{i}=1 \quad \lambda_{i} \geq 0, \quad i=1, \ldots, L
$$

At each time $k$, the sequence of future controls $U_{\infty}=$ $\{u(k \mid k), u(k+1 \mid k), u(k+2 \mid k), \ldots\}$ is obtained as solution of the following min-max optimization problem:

$$
\min _{U_{\infty}} \max _{(A, B) \in \Omega} J_{\infty}\left(A, B, U_{\infty}\right)
$$

where

$$
J_{\infty}\left(A, B, U_{\infty}\right)=\sum_{j=0}^{\infty}\left[\|x(k+j \mid k)\|_{W_{x}}^{2}+\|u(k+j \mid k)\|_{W_{u}}^{2}\right]
$$

in which $W_{x}>0$ and $W_{u}>0$ are symmetric weighting matrices. By assumption, all states are available for feedback so that $x(k \mid k)=x(k)$.
This min-max problem can be replaced with the following convex optimization problem with variables $\gamma \in \mathbb{R}$, $Q \in \mathbb{R}^{n \times n}, \Sigma \in \mathbb{R}^{p \times n}$, and LMI constraints $[2,7]$ :

$$
\min _{\gamma, Q, \Sigma} \gamma
$$

subject to $\left[\begin{array}{cc}Q & x(k) \\ \cdot & 1\end{array}\right]>0$,

$$
\left[\begin{array}{cccc}
Q & 0 & 0 & A_{i} Q+B_{i} \Sigma \\
\cdot & \gamma I & 0 & W_{x}^{1 / 2} Q \\
\cdot & \cdot & \gamma I & W_{u}^{1 / 2} \Sigma \\
\cdot & \cdot & \cdot & Q
\end{array}\right]>0, \quad i=1,2, \ldots, L
$$

If the problem (6)-(8) has a solution $\gamma_{k}^{*}, Q_{k}^{*}, \Sigma_{k}^{*}$, then the optimal control sequence is given by

$$
u(k+j \mid k)=K_{k}^{*} x(k+j \mid k),
$$

where

$$
K_{k}^{*}=\Sigma_{k}^{*}\left(Q_{k}^{*}\right)^{-1}
$$

Symmetric constraints on the manipulated variables of the form $\left|u_{l}(k+j \mid k)\right|<\bar{u}_{l}, l=1,2, \ldots, p, j \geq 0$ and on the output variables of the form $\left|y_{m}(k+j+1 \mid k)\right|<\bar{y}_{m}, m=$ $1,2, \ldots, q, j \geq 0$ can be imposed by including additional LMIs [7]

$$
\left[\begin{array}{ll}
X & \Sigma \\
\cdot & Q
\end{array}\right]>0
$$

with

$$
\begin{aligned}
& X_{l l}<\bar{u}^{2}, \quad l=1,2, \ldots, p, \\
& {\left[\begin{array}{cc}
Q & {\left[A_{i} Q+B_{i} \Sigma\right]^{T} C_{m}^{T}} \\
. & \bar{y}_{m}^{2}
\end{array}\right]>0, \quad i=1,2, \ldots, L}
\end{aligned}
$$

for $m=1,2, \ldots, q$, where $C_{m}$ denotes the $m$ th row of $C$.

Let $\mathbb{P}(x(k))$ denote the optimization problem (6) with constraints (7), (8), (11), and (12). Suppose that the control law uses the concept of receding horizon; that is, $u(k)=$ $K_{k}^{*} x(k)$ with $K_{k}^{*}$ recalculated at each sampling time $k$. The following lemma is concerned with convergence of the state trajectory to the origin under the constraints imposed on the operation of the plant.

Lemma 1. If $\mathbb{P}\left(x\left(k_{0}\right)\right)$ is feasible at some time $k=k_{0}$, then $\|x(k)\| \rightarrow 0$ as $k \rightarrow \infty$ with satisfaction of the input and output constraints.

The proof of Lemma 1 follows directly from the recursive feasibility and asymptotic stability properties demonstrated in [7]. 
Remark 2. As shown in Appendix A of Kothare et al. [7], $\mathbb{P}(x(k))$ is equivalent to minimizing a function $V(x(k \mid k))=$ $x(k \mid k)^{T} P_{k} x(k \mid k)$ with $P_{k}=\gamma_{k}^{*}\left(Q_{k}^{*}\right)^{-1}>0$. Such $V(x(k \mid$ $k)$ ) function is found to be an upper bound for the cost function $J_{\infty}\left(A, B, U_{\infty}\right)$ in $(5)$ and can be used as a candidate Lyapunov function in the proof of asymptotic stability.

Remark 3. For a regulation problem around a point different from the origin, a change of variables can be used, so that the new origin corresponds to the desired equilibrium point [7]. In the present work, the new value for the reference signal will be termed a pseudoreference. It is assumed that the process is integrating, and, therefore, the control value in steady state $\left(u_{s s}\right)$ is zero for any value of the pseudoreference. Otherwise, the determination of $u_{\mathrm{ss}}$ would not be trivial since the system matrices $A$ and $B$ are subjected to model uncertainties.

Remark 4. The RMPC problem formulation presented in this section considers that the matrices $A$ and $B$ are unknown but dot not vary with time. This is a particular case of the general framework introduced in [7], which was concerned with time-varying matrices $A(k)$ and $B(k)$.

\section{Treatment of Asymmetric Constraints}

The present work is concerned with regulation problems around the origin involving a SISO system with output variable $y(k)=C x(k)$. This is a particular case of the problem described in Section 2, with $p=q=1$. Therefore, the indexes $l$ and $m$ in (12) can be omitted. Moreover, the system is assumed to be integrating, so that $u_{\mathrm{ss}}=0$ regardless of the pseudoreference, as discussed in Remark 3. It is also considered that the manipulated variable $u(k)$ is subjected to a symmetric constraint $\bar{u}$ as in Section 2. Suppose that the constraints on $y$ are of the following form:

$$
y_{\min }<y(k)<y_{\max }
$$

with $y_{\min }<0$ and $y_{\max }>0$. If $\left|y_{\min }\right|=y_{\max }$, then the symmetric constraint formulation presented in Section 2 can be applied directly by making $\bar{y}=y_{\max }$. If $\left|y_{\min }\right| \neq y_{\max }$, a different approach is required. One alternative is to adopt a more conservative constraint, that is,

$$
\bar{y}= \begin{cases}y_{\max }, & \text { if } y_{\max }<\left|y_{\min }\right| \\ -y_{\min }, & \text { if } y_{\max }>\left|y_{\min }\right| .\end{cases}
$$

However, this procedure may not be convenient in the following cases:

(i) $y_{\max }<\left|y_{\min }\right|$ with $y_{\min } \leq y(k)<-y_{\max }$,

(ii) $y_{\max }>\left|y_{\min }\right|$ with $-y_{\min }<y(k) \leq y_{\max }$.

In these cases, the initial value of the output is admissible under the original asymmetric constraints, but not under the more conservative constraint (14). Figure 1(a) provides an illustration for case (i). As can be seen, the imposition of the more conservative constraint $-y_{\max }<y(k)<y_{\max }$ makes the output variable $y(k)$ be located outside of the range of admissible values.

Cavalca et al. [4] proposed a heuristic solution, based on a time-varying pseudoreference $r(k)=\min \left\{\left(y_{\max }+y(k)\right) /\right.$ $2,0\}$ as shown in Figure 1(b). The problem of asymmetric output constraints is then redefined in terms of new symmetric constraints $(a / a)$ around $r(k)$. However, it should be noted that this technique does not lead to guaranteed stability and constraint satisfaction.

Unlike the approach described above, which involves a pseudoreference $r(k)$ that may change at each sampling time $k$, the solution proposed in the present paper employs a sequence of pseudoreferences $r_{i}\left(i=1,2, \ldots, i_{\max }\right)$ which are defined at $k=0$ on the basis of the initial output value $y(0)$. As illustrated in Figure 2, symmetric constraints $(a / a, b / b, c / c, \ldots)$ are established around each pseudoreference. It will be shown that the use of such pseudoreferences, together with a convenient commutation rule, provides robust constraint satisfaction and ensures that the state trajectory converges to the origin. For sake of brevity of presentation, only case (i) will be treated. Case (ii) can be recast into case (i) by defining $\breve{y}(k)=-C x(k)$ and replacing constraints $y_{\min } \leq y(k) \leq y_{\max }$ with $-y_{\max } \leq \breve{y}(k) \leq-y_{\min }$.

Given an initial state $x(0)$ such that $y(0)=C x(0)$ falls within the scope of case (i), the following algorithm defines the pseudoreferences $r_{i}$, as well as a sequence of associated matrices $\widetilde{Q}_{i}^{*}$. These matrices will be subsequently employed in the control law to establish a rule of commutation from one pseudoreference to the next.

It is assumed that the set $X_{s}$ of possible equilibrium values $x_{s}$ for the state of the plant is known from the physics of the process to be controlled.

Algorithm for Determination of the Pseudoreferences and Associated Ellipsoids (PR Algorithm).

Step 1. Define the pseudoreferences $r_{i}$ as follows:

$$
\begin{aligned}
& \text { Step 1.1. Let } r_{0}=\left[y_{\max }+y(0)\right] / 2 \\
& \text { Step 1.2. Let } i=1 \\
& \text { Step 1.3. While }\left|r_{i-1}\right|>y_{\max } \text { do } \\
& \qquad \begin{array}{r}
r_{i}=\left(y_{\max }+r_{i-1}\right) / 2 \\
i=i+1
\end{array}
\end{aligned}
$$

End While

Step 1.4. Let $i_{\max }=i$

Step 1.5. Let $r_{i_{\max }}=0$.

Step 2. For each $r_{i}$ determine $x_{s, i}$ such that:

$$
\begin{gathered}
C x_{s, i}=r_{i}, \\
x_{s, i} \in X_{s} .
\end{gathered}
$$




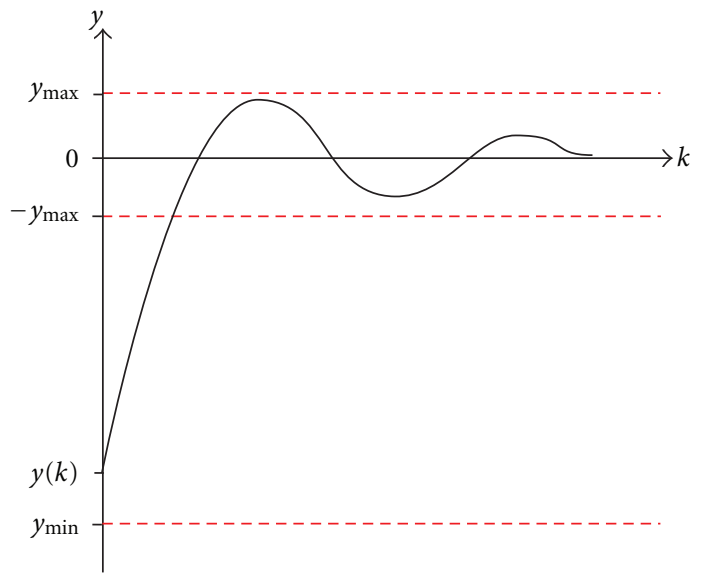

(a)

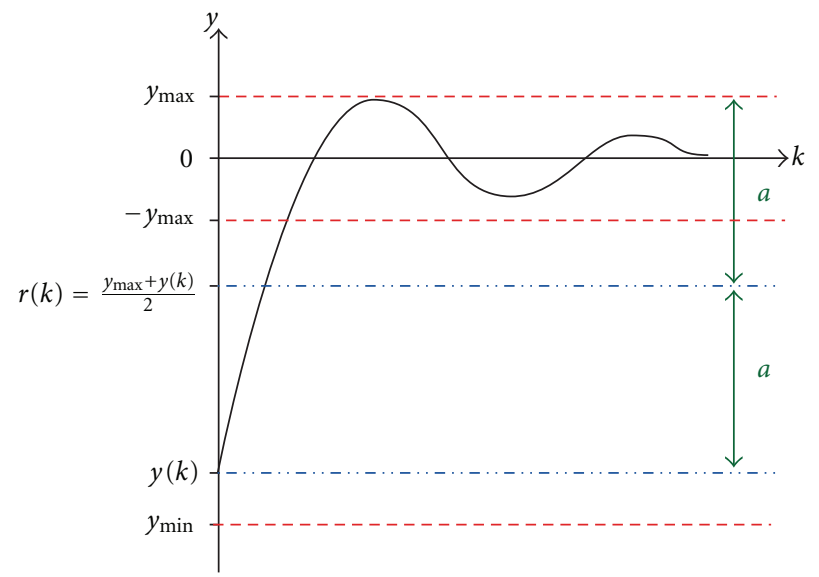

(b)

FIGURE 1: (a) Illustration of the more conservative constraint (14) in case (i). (b) Heuristic solution proposed by Cavalca et al. [4].

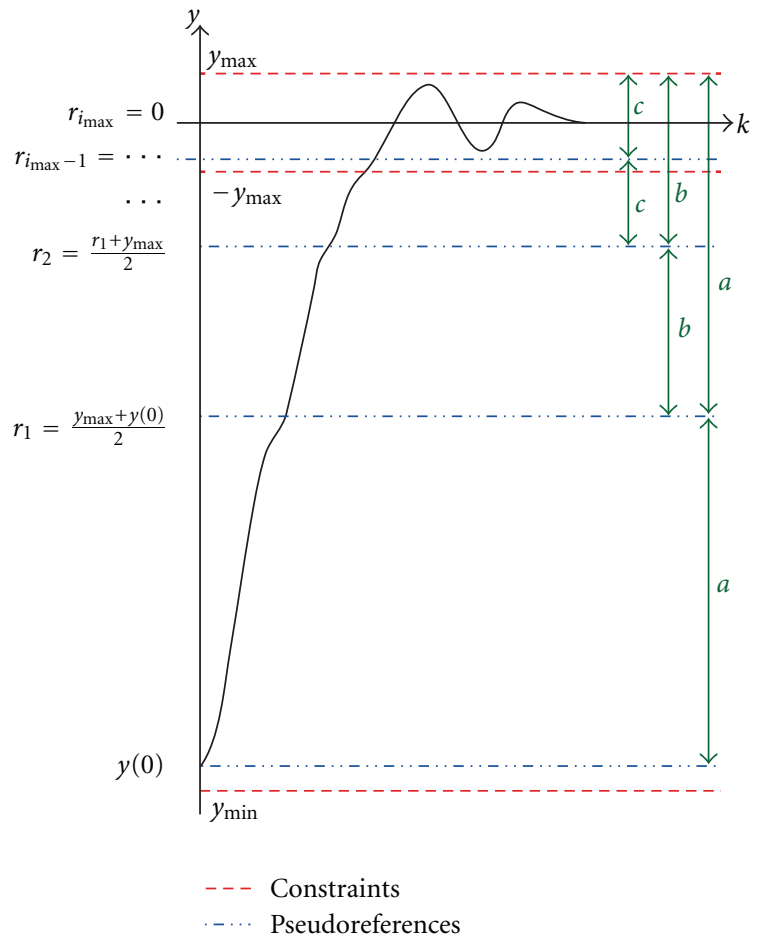

FIGURE 2: Illustration of the proposed pseudoreference scheme.

Step 3. Let

$$
\begin{array}{ll}
\xi_{0}=x(0)-x_{s, 0} & \\
\xi_{i}=x_{s, i-1}-x_{s, i}, & 1 \leq i \leq i_{\max } \\
\overline{y_{i}}=y_{\max }-r_{i}, & 0 \leq i \leq i_{\max } .
\end{array}
$$

Step 4. Solve the problem $\mathbb{P}\left(\xi_{i}\right)$ with the constraints $\overline{y_{i}}, \bar{u}$ for each $i\left(i=0,1, \ldots, i_{\max }\right)$ and denote the resulting solution by $\left(\tilde{\gamma}_{i}^{*}, \widetilde{Q}_{i}^{*}, \tilde{\Sigma}_{i}^{*}\right)$.

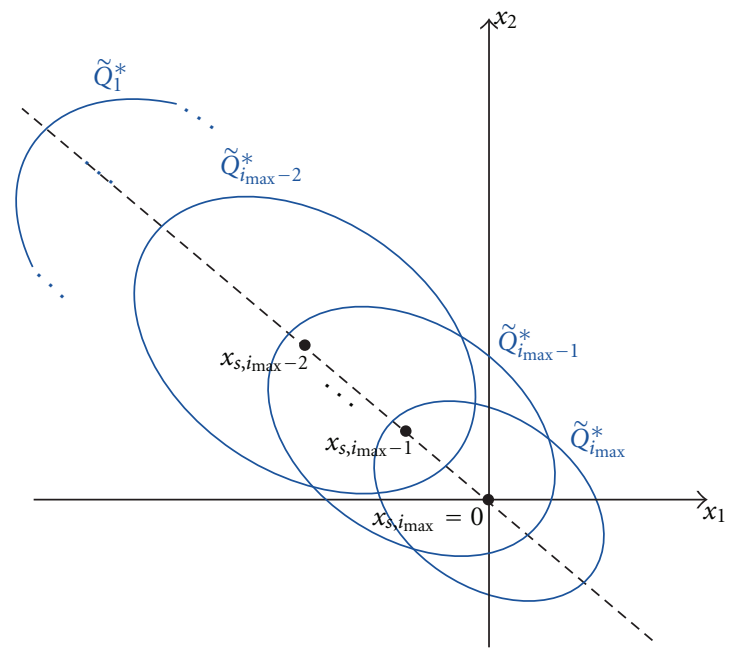

FIgURE 3: Result of the PR algorithm.

The matrices $\widetilde{Q}_{i}^{*}$ obtained in Step 4 define ellipsoids of the form $\left(x-x_{s, i}\right)^{T}\left(\tilde{Q}_{i}^{*}\right)^{-1}\left(x-x_{s, i}\right)<1$, as illustrated in Figure 3 for the case of a second-order system. It is noteworthy that, by construction (in view of LMI (7) with $\left.x(k)=\xi_{i}\right)$, the ellipsoid $i$ contains the center of the ellipsoid $(i-1)$.

The PR algorithm is said to be feasible if (15) and (16) in Step 2 and the optimization problem $\mathbb{P}\left(\xi_{i}\right)$ in Step 4 are feasible for every $i=1,2, \ldots, i_{\max }$. In this case, the resulting $x_{s, i}, \overline{y_{i}}, \widetilde{Q}_{i}^{*}, i=0,1, \ldots, i_{\max }$ are used in the control algorithm proposed below.

Algorithm for Control Using the Pseudoreferences (CPR Algorithm).

Initialization. Let $k=0$ and $i=0$.

Step 1. Read the state $x(k)$. 
Step 2. If $i<i_{\max }$ then

$$
\begin{aligned}
& \text { Let } \tilde{x}_{i+1}(k)=x(k)-x_{i+1, s} \\
& \text { If } \tilde{x}_{i+1}^{T}(k)\left(\tilde{Q}_{i+1}^{*}\right)^{-1} \tilde{x}_{i+1}(k)<1 \text { (condition of transi- } \\
& \text { tion) then }
\end{aligned}
$$

$$
\text { Let } i=i+1
$$

End If

End If.

Step 3. Let $\tilde{x}_{i}(k)=x(k)-x_{s, i}$ and solve the problem $\mathbb{P}\left(\widetilde{x}_{i}(k)\right)$ with the constraints $\overline{y_{i}}$ and $\bar{u}$ in order to obtain $\left(\gamma_{k}^{*}, Q_{k}^{*}, \Sigma_{k}^{*}\right)$.

Step 4. Calculate $K_{k}^{*}=\Sigma_{k}^{*}\left(Q_{k}^{*}\right)^{-1}$ and $u(k)=K_{k}^{*} \tilde{x}_{i}(k)$.

Step 5. Apply $u(k)$ to the plant.

Step 6. Let $k=k+1$, wait a sample time and return to Step 1 .

The main result of this work is stated in the following theorem, which is concerned with the satisfaction of constraints and convergence of the state trajectory to the origin under the control law given by the CPR algorithm.

Theorem 5. If the $P R$ algorithm is feasible and the $C P R$ algorithm is applied to control the plant, then $\|x(k)\| \rightarrow 0$ as $k \rightarrow \infty$, with satisfaction of the input and output constraints.

Proof. For $k=0$, the state $x(k)$ lies in the ellipsoid associated with $\widetilde{Q}_{0}^{*}$, which was obtained by solving problem $\mathbb{P}(x(0)-$ $\left.x_{s, 0}\right)$ in the PR algorithm. Therefore, problem $\mathbb{P}\left(\tilde{x}_{0}(0)\right)$ is feasible by hypothesis. Lemma 1 then guarantees that $\left\|\tilde{x}_{0}(k)\right\| \rightarrow 0$ (i.e., $\left\|x(k)-x_{s, 0}\right\| \rightarrow 0$ ) as $k \rightarrow \infty$, with satisfaction of the input and output constraints, under the control law stated in Steps 3 and 4 of the CPR algorithm with $i=0$. Since the ellipsoid associated with $\widetilde{Q}_{1}^{*}$ contains $x_{s, 0}$, by construction, it can be concluded that the condition of transition stated in Step 2 of the CPR algorithm with $i=1$ will be satisfied in finite time. Let $k_{1}$ be the first time when this condition is satisfied, that is,

$$
\tilde{x}_{1}^{T}\left(k_{1}\right)\left(\widetilde{Q}_{1}^{*}\right)^{-1} \tilde{x}_{1}\left(k_{1}\right)<1 .
$$

This condition ensures that the optimization problem $\mathbb{P}\left(\tilde{x}_{1}\left(k_{1}\right)\right)$ is feasible, since, by construction, the solution $\left(\tilde{\gamma}_{1}^{*}, \widetilde{Q}_{1}^{*}, \tilde{\Sigma}_{1}^{*}\right)$ of $\mathbb{P}\left(\xi_{1}\right)$ satisfies the constraints of $\mathbb{P}\left(\tilde{x}_{1}\left(k_{1}\right)\right)$. In fact, condition (17) ensures that LMI (7) is satisfied with $x(k)$ and $Q$ replaced with $\widetilde{x}_{1}\left(k_{1}\right)$ and $\widetilde{Q}_{1}^{*}$, respectively. Moreover, the remaining LMIs (8),(11)-(12) are satisfied by $\widetilde{Q}_{1}^{*}$ and $\widetilde{\Sigma}_{1}^{*}$ by hypothesis. Therefore, after switching from $i=0$ to $i=1$, Lemma 1 ensures that $\left\|\tilde{x}_{1}(k)\right\| \rightarrow 0$ (i.e., $\left\|x(k)-x_{s, 1}\right\| \rightarrow 0$ ) as $k \rightarrow \infty$ with satisfaction of the input and output constraints. As a result, the condition of transition with $i=1$ will be satisfied in finite time. A similar reasoning can be applied to show that the condition of transition will be satisfied for all $i=0,1, \ldots, i_{\max }-1$ in finite time. Finally, when $i=i_{\max }$, the state $x(k)$ will be inside the last ellipsoid, centered at $x_{s, i_{\max }}=\left[\begin{array}{llll}0 & 0 & \cdots & 0\end{array}\right]^{T}$, and then

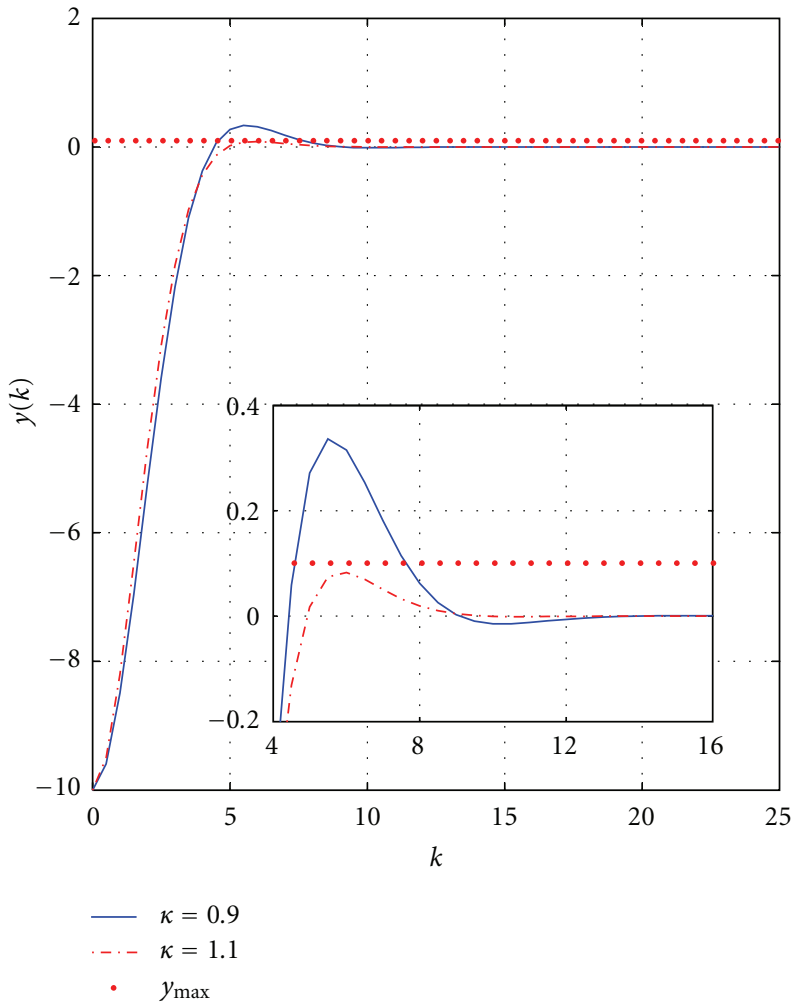

FIgURE 4: Simulation results using relaxed output constraints.

Lemma 1 will ensure that $\|x(k)\| \rightarrow 0$ as $k \rightarrow \infty$, again with satisfaction of the constraints.

\section{Numerical Example}

A discrete state-space model of a double integrator will be employed to illustrate the proposed strategy. The matrices of the model are given by

$$
A=\left[\begin{array}{ll}
1 & T \\
0 & 1
\end{array}\right], \quad B=\left[\begin{array}{c}
T^{2} / 2 \\
T
\end{array}\right] \kappa, \quad C=\left[\begin{array}{ll}
1 & 0.2
\end{array}\right],
$$

where $T$ is the sampling time, and $\kappa$ is an uncertain gain parameter.

The initial condition is set to $x(0)=\left[-100^{T}\right]$, the sampling time is $T=0.5 \mathrm{~s}$, and the constraints are defined as $\bar{u}=u_{\max }=u_{\min }=5, y_{\min }=-10$ and $y_{\max }=0.1$. The uncertain parameter $\kappa$ is assumed to be in the range $0.9-1.1$. The weight matrices of the controller are defined as

$$
W_{x}=\left[\begin{array}{cc}
100 & 0 \\
0 & 0.01
\end{array}\right], \quad W_{u}=100 .
$$

In this case, the set $X_{s}$ of possible equilibrium points is given by

$$
X_{s}=\left\{\left(x_{1}, x_{2}\right) \mid x_{2}=0\right\} .
$$




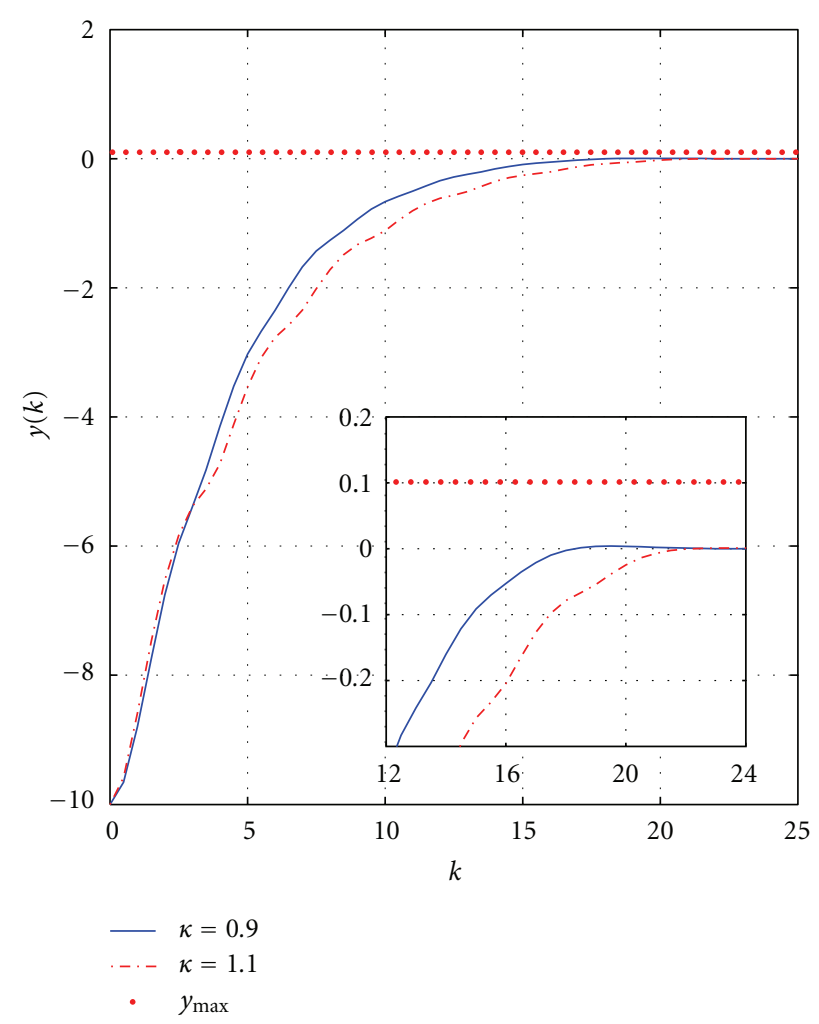

(a)

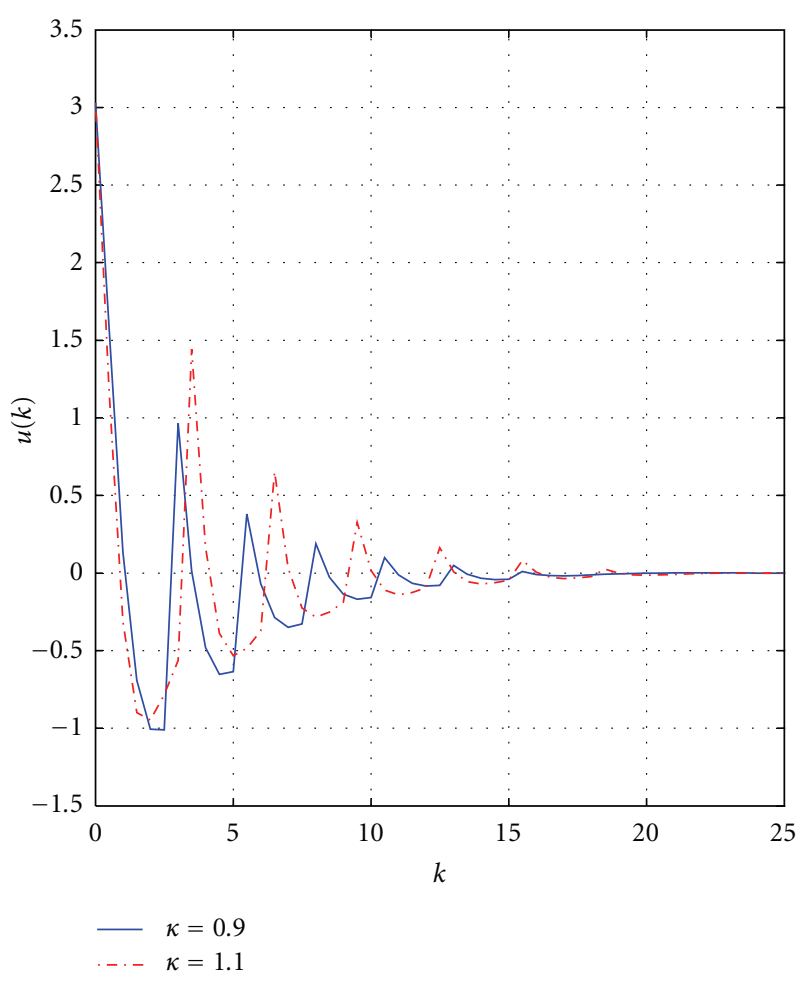

(b)

FIGURE 5: Simulation results using the proposed strategy: (a) output and (b) control signals.

TABLE 1: Pseudoreferences and associated output constraints.

\begin{tabular}{ccc}
\hline$i$ & $r_{i}$ & $\overline{y_{i}}$ \\
\hline 0 & -4.95 & 5.05 \\
1 & -2.425 & 2.525 \\
2 & -1.1625 & 1.2625 \\
3 & -0.53125 & 0.63125 \\
4 & -0.21563 & 0.31562 \\
5 & -0.057813 & 0.15781 \\
6 & 0 & 0.1 \\
\hline
\end{tabular}

In fact, the state variables $x_{1}$ and $x_{2}$ can be regarded as position and velocity, respectively, and thus the equilibrium can only be achieved if the velocity $x_{2}$ is zero.

The pseudoreferences $r_{i}$ and associated symmetric output constraints $\overline{y_{i}}$, which define the controllers $i=0, \ldots, 6$, are presented in Table 1 . The simulations were performed in the Matlab environment.

\section{Results and Discussions}

As discussed in Section 3, a possible approach to address asymmetric constraints consists of adopting the conservative bounds defined in (14). In the present example, such procedure amounts to setting $\bar{y}=y_{\max }=0.1$. However, the resulting optimization problem $\mathbb{P}(x(0))$ becomes infeasible. In fact, given the control constraint $-5<u(k)<5$, it is not possible to steer the output from $y(0)=-10$ to the range $[-0.1,0.1]$ in a single sampling period.

On the other hand, if the constraints are relaxed by setting $\bar{y}=-y_{\min }=10$, there is no guarantee that the resulting output trajectory will remain within the original $\left(y_{\min }, y_{\max }\right)$ bounds. In fact, the inset in Figure 4 shows that such a relaxation of the output constraints does lead to a violation of the original upper bound for $\kappa=0.9$.

These findings motivate the adoption of the proposed strategy for handling the asymmetric output constraints. Figures 5(a) and 5(b) present the simulation results obtained by using the CPR algorithm. As can be seen, both the output and control constraints were properly enforced, even by using the extreme values of $\kappa$ in the simulation.

The commutation between the successive pseudoreferences is illustrated in Figure 6. This graph indicates that the commutation from one pseudoreference to the next occurs in finite time, as expected. The final pseudoreference $(i=6)$ corresponds to the origin, which is the desired equilibrium point for the regulation problem.

\section{Conclusion}

This paper presented a strategy for handling asymmetric output constraints within the scope of an LMI-based RMPC 


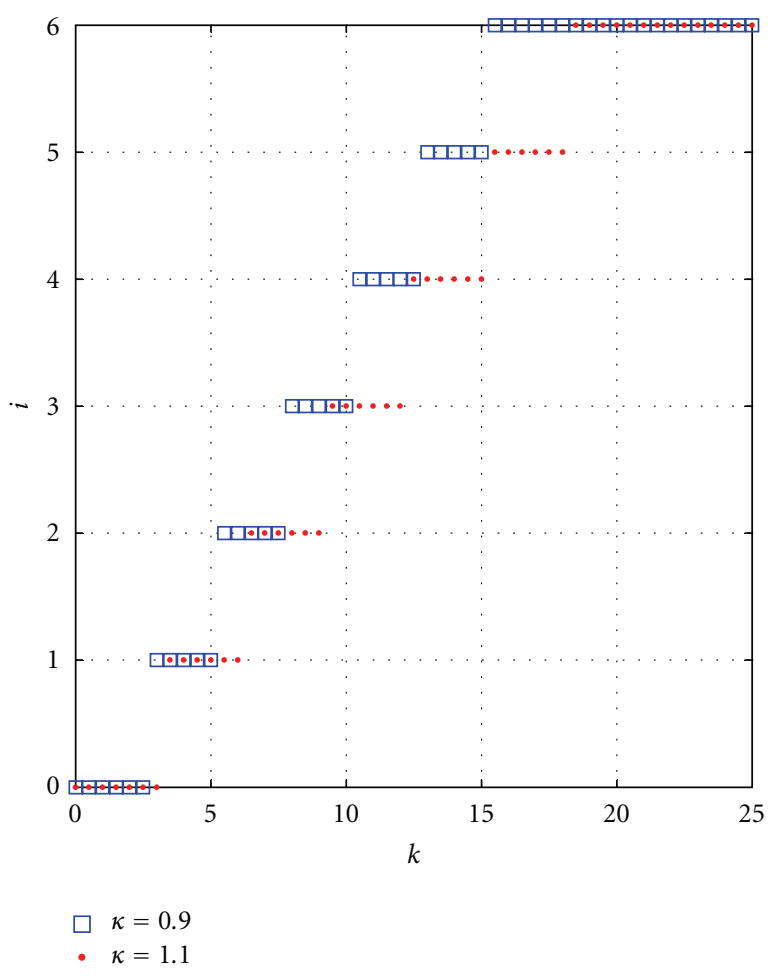

Figure 6: Commutation between pseudoreferences.

scheme. For this purpose, a procedure for defining a sequence of pseudoreferences was devised, along with a rule for commutation from one pseudoreference to the next. The proposed approach guarantees constraint satisfaction and convergence of the state trajectory to the origin, provided that the algorithm for determination of the pseudoreferences is feasible. The results of a numerical simulation study indicated that the proposed procedure may be a suitable alternative to the use of either more conservative constraints (which may lead to infeasibility issues) or more relaxed constraints (which do not guarantee satisfaction of the original restrictions). Future research could be concerned with the extension of the proposed approach to multiple input-multiple output (MIMO) systems. In this case, it may be necessary to define different pseudoreferences for each constrained output under consideration.

\section{Acknowledgments}

The authors gratefully acknowledge the support of FAPESP (scholarship 2008/54708-6 and grant 2006/58850-6), CAPES (Pró-Engenharias), and $\mathrm{CNPq}$ (research fellowships).

\section{References}

[1] E. F. Camacho and C. Bordons, Model Predictive Control, Spring, London, UK, 1999.

[2] J. M. Maciejowski, Predictive Control with Constraints, Prentice-Hall, Harlow, UK, 2002.

[3] J. A. Rossiter, Model-based predictive control-a practical approach, CRC Press, New York, NY, USA, 2003.
[4] M. S. M. Cavalca, R. K. H. Galvão, and T. Yoneyama, "Robust model predictive control for a magnetic levitation system employing linear matrix inequalities," in ABCM Symposium Series in Mechatronics, vol. 4, Rio de Janeiro, Brazil, 2010.

[5] A. H. González, J. L. Marchetti, and D. Odloak, "Robust model predictive control with zone control," IET Control Theory and Applications, vol. 3, no. 1, pp. 121-135, 2009.

[6] M. A. Rodrigues and D. Odloak, "Output feedback MPC with guaranteed robust stability," Journal of Process Control, vol. 10, no. 6, pp. 557-572, 2000.

[7] M. V. Kothare, V. Balakrishnan, and M. Morari, "Robust constrained model predictive control using linear matrix inequalities," Automatica, vol. 32, no. 10, pp. 1361-1379, 1996.

[8] L. Özkan and M. V. Kothare, "Stability analysis of a multimodel predictive control algorithm with application to control of chemical reactors," Journal of Process Control, vol. 16, no. 2, pp. 81-90, 2006.

[9] Z. Wan and M. V. Kothare, "A framework for design of scheduled output feedback model predictive control," Journal of Process Control, vol. 18, no. 3-4, pp. 391-398, 2008.

[10] V. T. Minh and F. B. M. Hashim, "Robust model predictive control schemes for tracking setpoints," Journal of Control Science and Engineering, vol. 2010, Article ID 6494361, 2010.

[11] M. S. M. Cavalca, R. K. H. Galvão, and T. Yoneyama, "Integrator resetting with guaranteed feasibility for an LMI-based robust model predictive control approach," in Proceedings of the 18th Mediterranean Conference on Control and Automation, pp. 634-639, Marrakech, Morocco, 2010.

[12] Z. Wan and M. V. Kothare, "An efficient off-line formulation of robust model predictive control using linear matrix inequalities," Automatica, vol. 39, no. 5, pp. 837-846, 2003.

[13] Z. Wan and M. V. Kothare, "Robust output feedback model predictive control using off-line linear matrix inequalities," Journal of Process Control, vol. 12, no. 7, pp. 763-774, 2002. 

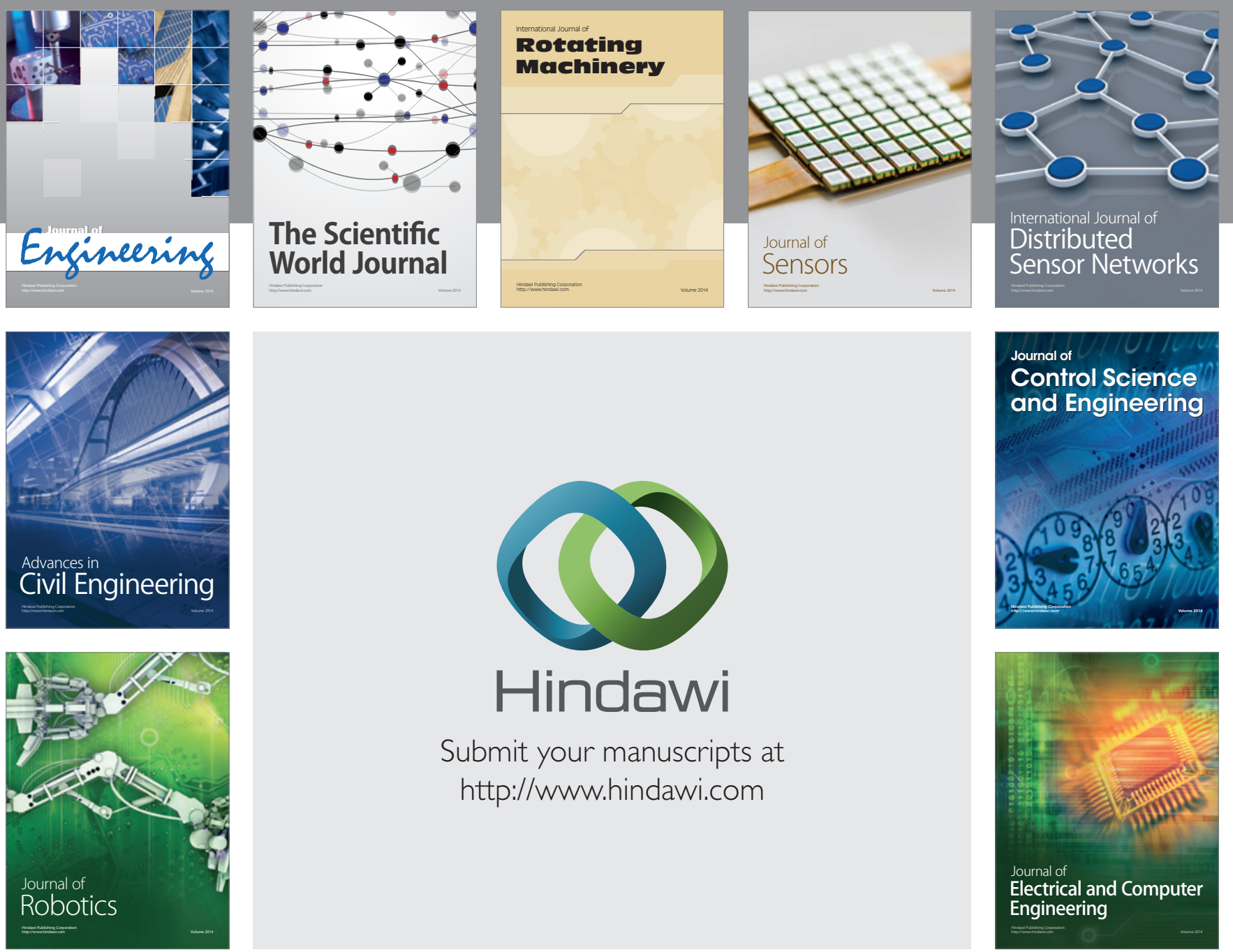

Submit your manuscripts at

http://www.hindawi.com
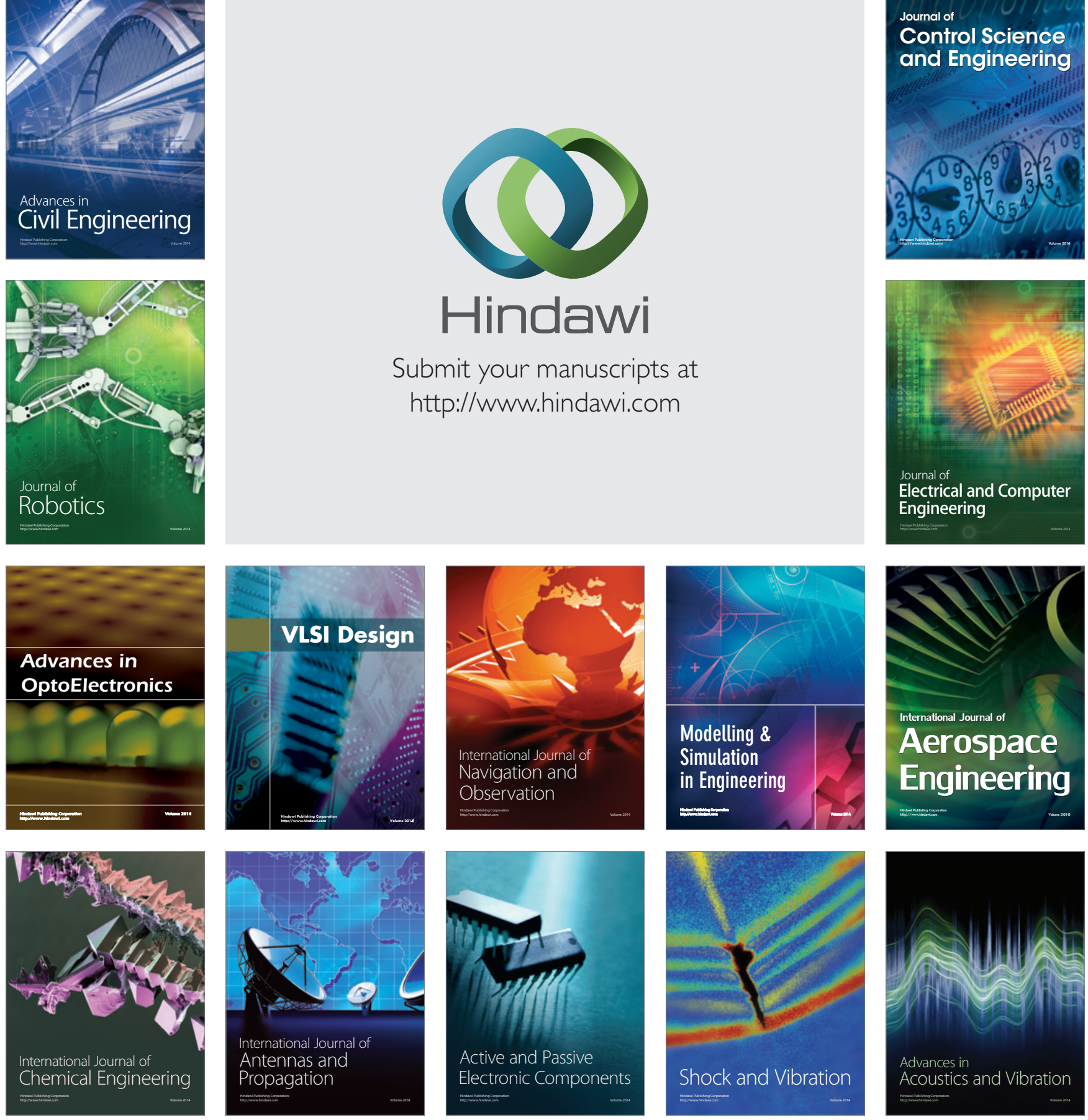